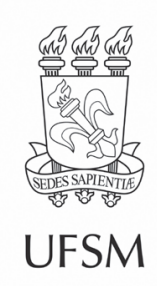

\title{
Artigos
}

\section{Dinâmica do componente arbóreo e regenerante em uma floresta nebular no Planalto Sul Catarinense}

\author{
Dynamics of adult and regenerative tree species components in a cloud \\ forest in the South Plateau of Santa Catarina state, Brazil
}

\begin{abstract}
Guilherme Neto Santos ${ }^{\oplus}$, Ana Carolina da Silva ${ }^{\oplus}$, Pedro Higuchi' ${ }^{\oplus}$, Aline Gross ${ }^{\oplus}$, Ricardo de Vargas Kilca ${ }^{\oplus}$, Mariele Alves Ferrer Silva ${ }^{\oplus}$, Carla Luciane Lima ${ }^{\oplus}$, Aline Pereira $\mathrm{Cruz}^{1}{ }^{\oplus}$, Amanda da Silva Nunes ${ }^{\oplus}$, Vanessa Fátima Soboleski' ${ }^{\oplus}$
\end{abstract}

'Universidade do Estado de Santa Catarina, Lages, SC, Brasil

\section{RESUMO}

Avaliou-se a dinâmica do componente arbóreo e regenerante em uma floresta nebular, na região do Planalto Sul Catarinense, com o objetivo de se conhecer o processo de desenvolvimento da vegetação ao longo do tempo. Para isso, foram instaladas parcelas permanentes onde foram caracterizados os componentes adulto e regenerante, respectivamente, nos anos de 2011 e 2013. Todas as espécies amostradas foram classificadas quanto à guilda de regeneração em pioneiras, climácicas exigentes em luz e climácicas tolerantes ao sombreamemto. No ano de 2015, foi realizado o segundo inventário dos componentes vegetacionais, sendo determinado o crescimento dos sobreviventes (somente adultos) e contabilizados os indivíduos recrutados e mortos. Para cada uma das populações, foram determinadas as taxas de mudança líquida em número de indivíduos (adultos e regenerantes) e em área basal (adultos). Foram realizados testes de proporções para verificar se a representatividade das diferentes guildas de regeneração se alterou entre os anos avaliados. Os resultados indicaram que a floresta se encontra em um processo de estruturação, caracterizado pelo ganho em número de indivíduos e em área basal. Por outro lado, observou-se estabilidade na riqueza e na representatividade das guildas de regeneração, com destaque para a maior participação de espécies classificadas como clímax exigentes em luz. Conclui-se que a floresta avaliada se encontra em estruturação, com o favorecimento da regeneração de espécies exigentes em luz.

Palavras-chave: Floresta Ombrófila Mista Alto-Montana; Guildas de regeneração; Taxas demográficas 


\begin{abstract}
The dynamics of adult and regenerative tree species components was assessed in a cloud forest in the South Plateau of Santa Catarina state, southern Brazil, seeking a better understanding of the process of vegetation development. To this end, permanent sampling plots were allocated, where the adult and regenerative components were characterized, respectively, in 2011 and 2013. All surveyed species were classified according to regeneration guilds into pioneer, light-demanding climax, and shade-tolerant climax. In 2015, a second tree species component survey was conducted to determine the growth of surviving individuals (adults) and count the recruited and dead individuals. For each population, the net change rates for individuals (adults and regenerative) and basal area (only adults) were determined. Proportion tests were conducted to verify whether representativeness of the regeneration guilds changed over the study period. The results showed that the forest investigated is undergoing a structuring process characterized by gains in number of individuals and basal area. In contrast, stability in species richness and representativeness of regeneration guilds was verified, with highlight for lightdemanding climax species. In conclusion, the assessed forest is undergoing a structuring phase that favors the regeneration of light-demanding species.
\end{abstract}

Keywords: Upper montane Araucaria Forest; Regeneration guilds; Demographic rates

\title{
1 INTRODUÇÃO
}

No sul do Brasil, em locais cuja altitude aproximada é de 1.300 m, a Floresta Ombrófila Mista Alto-Montana encontra-se em um ambiente caracterizado pela nebulosidade constante e baixas temperaturas, podendo ser denominada como floresta nebular (BERTONCELLO et al., 2011). Essas condições ambientais criam um ambiente peculiar e seletivo, em que há redução nas taxas de decomposição da biomassa e crescimento reduzido das espécies arbóreas, imprimindo características florístico-estruturais únicas no componente arbóreo (HIGUCHI et al., 2012). Apesar de serem particularmente sensíveis a possíveis impactos de mudanças climáticas e estarem sujeitas a perturbações antrópicas crônicas, as florestas nebulares apresentam elevada importância ecológica por desempenharem importantes funções ambientais, como a proteção de nascentes e bacias hidrográficas (HAMILTON; JUVIK; SCATENA, 1995; SCHEER; MOCOCHINSK, 2009), assim como a manutenção de um ecossistema único com ocorrência de espécies endêmicas.

No Planalto Catarinense, as florestas regionais foram sujeitas ao "ciclo da 
madeira", caracterizado pela exploração intensa das florestas com araucária, que se iniciou na década de 1910, com a construção da ferrovia que ligava São Paulo ao Rio Grande do Sul (CARVALHO; NODARI, 2008; FERREIRA, 2009). Em meados do século, a indústria obteve sua maior expansão, atingiu seu auge, e, segundo Carvalho e Nodari (2008), em 1970, já se observavam indícios do esgotamento das florestas. Dessa forma, os fragmentos atuais de Floresta Ombrófila Mista (FOM) são remanescentes desse período e sofreram cortes rasos ou seletivos no passado. Em FOM Alto-Montanas, a exploração também foi intensa e foi seguida da criação de gado nas pastagens formadas a partir do desmatamento e em campos naturais adjacentes.

Dessa forma, acompanhar o desenvolvimento da composição arbórea nessas áreas é de elevada importância para prever os impactos que podem ocorrer sobre a vegetação. Assim, estudos de dinâmica florestal se tornam cada vez mais importantes, pois permitem obter informações das taxas de crescimento e mortalidade, assim como das mudanças que ocorrem na composição florística e estrutural de um componente arbóreo. Esses estudos geram informações que podem contribuir para a definição de estratégias de recuperação que possam minimizar os impactos ocorridos, assim como para a aplicação de técnicas de manejo visando à conservação dos remanescentes e ao uso sustentável dos recursos florestais (SHEIL; JENNINGS; SAVILL, 2000; SANTOS et al., 2012).

As taxas demográficas (e.g., mortalidade, recrutamento, ganho e perda em área basal) obtidas a partir dos estudos de dinâmica têm apresentado muitas variações de acordo com a floresta estudada (LIEBERMAN; LIEBERMAN, 1987), sendo que pouco se conhece sobre floresta nebular, o que torna maior a necessidade desses estudos, facilitando o planejamento de estratégias de conservação (HIGUCHI et al., 2008). Para a Floresta Ombrófila Mista, em escala regional, se a influência da altitude tem sido evidenciada como um importante fator na distribuição de espécies arbóreas (HIGUCHI et al., 2012), para os padrões de dinâmica e de regeneração natural, o efeito da fragmentação florestal (GROSS et al., 2018) e os distúrbios crônicos (VEFAGO et al., 2019) 
se mostram mais importantes. Além disso, observa-se que, enquanto o componente regenerativo frequentemente apresenta um padrão de estruturação (i.e., aumento na abundância de regenerantes) ao longo do tempo (VEFAGO et al., 2019), o componente adulto frequentemente demonstra um padrão de autodesbaste, com a redução do número de árvores acompanhado pelo aumento da área basal (GROSS et al., 2018).

Sendo assim, o presente trabalho objetivou conhecer os padrões de dinâmica dos componentes adulto e regenerante de um fragmento de floresta nebular no Planalto Catarinense, a fim de obter resultados que auxiliem na previsão de possíveis mudanças florística e estruturais na comunidade e nas populações florestais. A hipótese é que a floresta se encontra em fase de estruturação, considerando o histórico de impacto sofrido durante períodos pretéritos de cortes seletivos.

\section{MATERIAL E MÉTODOS}

O fragmento florestal natural de floresta nebular estudado possui 346 ha e está localizado no município de Urubici, na Serra Catarinense, na latitude $28^{\circ} 04^{\prime}$ S e longitude $49^{\circ} 37^{\prime} \mathrm{O}$, com altitude aproximada de $1.600 \mathrm{~m}$. A região possui clima Cfb, de acordo com a classificação de Köppen, com temperatura média anual de 15 a $16^{\circ} \mathrm{C}$. A precipitação média anual varia entre 1.200 e 1.900 mm, com chuvas bem distribuídas durante o ano. A fitofisionomia do fragmento pode ser classificada, segundo a classificação do IBGE (2012), como Floresta Ombrófila Mista Alto-Montana, e a presença de nuvens constantes o caracteriza como uma área de floresta nebular.

O fragmento é pertencente a uma propriedade particular e está sob influência do gado, visto que este é encontrado nas pastagens adjacentes à área de estudo, possuindo acesso livre à floresta. Assim, são observados, especialmente na borda da floresta, alguns impactos causados pelos bovinos, como a presença de fezes dentro do fragmento. Apesar disso, a floresta se encontra bem estruturada, podendo ser classificada como de estágio sucessional avançado. 
A amostragem do fragmento foi realizada de forma sistemática, no ano de 2011, por Marcon et al. (2014), por meio de 25 unidades amostrais (parcelas), distanciadas $10 \mathrm{~m}$ entre si (Figura 1) de $400 \mathrm{~m}^{2}(20 \mathrm{~m} \times 20 \mathrm{~m})$ cada, para o estudo do componente arbóreo adulto (DAP - diâmetro medido à altura do peito $\geq 5 \mathrm{~cm}$ ). Dentro das parcelas, foram amostrados todos os indivíduos arbóreos vivos, sendo identificados por especialistas e com auxílio da literatura, plaqueteados e coletados os valores de CAP (circunferência à altura do peito), medidos com fita métrica, os quais posteriormente foram transformados em DAP. Indivíduos com troncos múltiplos foram medidos quando a raiz da soma dos quadrados dos DAPs foi maior do que $5 \mathrm{~cm}$. Em 2013, cada uma das 25 parcelas alocadas foi subdividida em subparcelas de diferentes tamanhos, para avaliação da regeneração natural realizada por Dalla Rosa et al. (2016). As subparcelas tiveram suas áreas estabelecidas de acordo com a classe de altura dos regenerantes, conforme metodologia utilizada por Volpato (1994): i) classe 1, plantas com altura a partir de $15 \mathrm{~cm}$ e até $1 \mathrm{~m}$, avaliadas em subparcelas de $5 \mathrm{~m}^{2}(5 \times 1 \mathrm{~m})$; ii) classe 2, plantas com altura maior que $1 \mathrm{~m}$ e até $3 \mathrm{~m}$, avaliadas em $10 \mathrm{~m}^{2}(10 \times 1 \mathrm{~m})$, e iii) classe 3, plantas com altura maior que $3 \mathrm{~m}$ e DAP menor que $5 \mathrm{~cm}$, avaliadas em $20 \mathrm{~m}^{2}(20 \times 1 \mathrm{~m})$. Assim, em cada subparcela, todos os indivíduos dentro das classes foram inventariados, sendo identificados por especialistas e com auxílio de literatura especializada, e plaqueteados.

No presente estudo, foi avaliada a demografia das populações amostradas em ambos os componentes (adulto e regenerante). Em 2015, quatro anos após o primeiro inventário do componente adulto e dois anos após o inventário do componente regenerante, foi realizado o segundo inventário de ambos os componentes, utilizandose as mesmas metodologias anteriores, no qual foram recrutados novos indivíduos que atingiram os valores de inclusão nas parcelas (5 cm de DAP) e subparcelas (15 cm de altura), sendo identificados, medidos (CAP dos adultos) ou classificados em classes (regenerantes), e plaqueteados. Os indivíduos sobreviventes foram mensurados 
novamente e os indivíduos mortos foram contabilizados.

Todas as espécies e indivíduos amostrados nos anos avaliados foram classificadas em guildas de regeneração, segundo a metodologia de Swaine e Whitmore (1988), adaptada por Oliveira Filho et al. (1994), em pioneira (P), clímax exigente de luz (CEL) ou clímax tolerante à sombra (CTS), com base nas observações em campo e consulta à literatura (LINDENMAIER; BUDKE, 2006; LEYSER et al., 2012; SANTOS et al., 2015). Duas espécies do componente adulto e uma do regenerante não foram classificadas em guildas, pois foram identificados somente em nível de gênero.

Figura 1 - Local de estudo para a caracterização da dinâmica das espécies arbóreas e regenerantes de um fragmento de floresta nebular no Planalto Catarinense

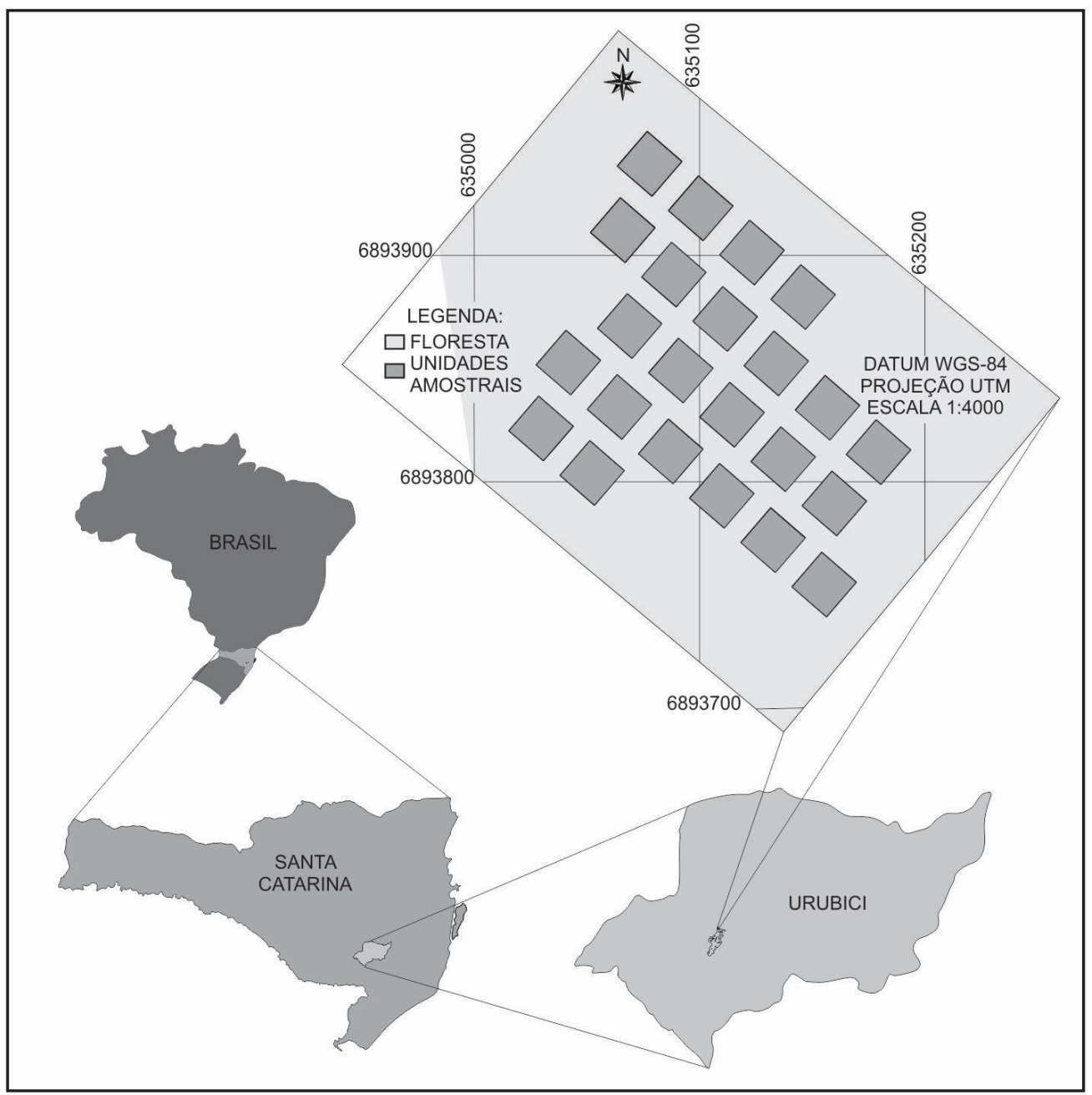

Fonte: Autores (2021) 
Para obtenção da demografia das espécies, foram calculadas as taxas de dinâmica de mudança líquida para o número de indivíduos $\left(\mathrm{ML}_{\mathrm{N}^{\prime}}\right.$ em \%.ano-1) (para ambos os componentes) e mudança líquida para a área basal $\left(\mathrm{ML}_{\mathrm{AB}^{\prime}}\right.$ em \%.ano-1) (componente adulto), pelas equações (1) e (2), conforme Korning e Balslev (1994):

$$
\begin{aligned}
& \text { MLn }=\left[\left(\mathrm{N}_{\mathrm{t}} / \mathrm{N}_{0}\right)^{\frac{1}{\mathrm{t}}}-1\right] * 100 \\
& \text { MLab }=\left[\left(\mathrm{AB}_{\mathrm{t}} / \mathrm{AB}_{0}\right)^{\frac{1}{\mathrm{t}}}-1\right] * 100
\end{aligned}
$$

Onde: $\mathrm{t}$ = intervalo de tempo entre os inventários; $\mathrm{N}_{\mathrm{o}}=$ número inicial de árvores; $\mathrm{N}_{\mathrm{t}}=$ número final de árvores depois de $t ; A B_{0}=$ área basal inicial $\left(m^{2} / h a\right) ; A B_{t}=$ área basal final depois de $t\left(m^{2} / h a\right)$.

Para a comparação entre os anos de avaliação das espécies e dos indivíduos nas guildas de regeneração, foi utilizado o teste de proporção, realizado no programa $R$ ( $R$ DEVELOPMENT CORE TEAM, 2017). Os demais cálculos foram realizados com auxílio da planilha eletrônica Excel.

\section{RESULTADOS E DISCUSSÃO}

A dinâmica estrutural do fragmento estudado foi caracterizada por maiores taxas de recrutamento do que de mortalidade, e de ganho do que de perda em área basal, resultando em mudanças líquidas positivas tanto para o número de indivíduos $\left(\mathrm{ML}_{\mathrm{N}}\right.$ adultos $=0,6 \%$.ano-1 e $\mathrm{ML}_{\mathrm{N}}$ regenerantes $=14,8 \%$.ano $\left.{ }^{-1}\right)$ quanto para a área basal $\left(\mathrm{ML}_{\mathrm{AB}}\right.$ adultos $=5,0 \%$.ano-1) $($ Tabela 1). Para o componente adulto, a taxa de mudança líquida em área basal (5,0 \%.ano-1) foi maior do que a taxa de mudança líquida em número de indivíduos $(0,6 \%$.ano-1) o que sugere que a estruturação da floresta se deu, principalmente, pelo incremento diamétrico das árvores sobreviventes. Destacase que, apesar de o número de indivíduos ter aumentado em ambos os componentes, as magnitudes dos valores observados diferiram, com os regenerantes apresentando maior incremento, de 526 para 604, do que os adultos, de 1.582 para 1.591. Tomados em conjunto, os resultados indicam que a floresta se encontra em um processo de estruturação, com destaque para o crescimento das árvores sobreviventes adultas 
e o incremento na densidade dos regenerantes. Esse aspecto da dinâmica sugere uma fase de "construção inicial" (LEITE; RODRIGUES, 2008), que ocorre em respostas a distúrbios pretéritos.

Nesse sentido, como também destacado por Salami et al. (2014), é possível inferir que a floresta, ao ganhar biomassa por meio do incremento do número de indivíduos e da área basal, esteja funcionando como um sumidouro de carbono atmosférico. Apesar de estudos sobre as mudanças demográficas da regeneração natural em condições de sub-bosque serem raros para a fitofisionomia avaliada, o que impede inferências comparativas, trabalhos sobre a dinâmica do componente adulto (e.g. SANQUETTA; DALLA CORTE; EISFELD, 2003; FORMENTO; SCHORN; RAMOS, 2004; SALAMI et al., 2014; GROSS et al., 2018) indicam que o padrão observado é comum na região. Por exemplo, Gross et al. (2018), em um estudo em áreas de Floresta Ombrófila Mista, considerando uma escala regional, observaram que, para um período de quatro anos, a área basal aumentou 0,79 \%. Considerando o histórico de perturbação da Floresta com Araucária no sul do Brasil, é possível inferir, a partir dos resultados encontrados no presente estudo e de outros trabalhos publicados, que a FOM se encontra em fase de recuperação estrutural após a intensa exploração predatória que ocorreu em meados do século passado, época conhecida como "ciclo da madeira".

Apesar do ganho em número de indivíduos para o componente regenerante e, especialmente em área basal, para os adultos, no que se refere à riqueza de espécies, observa-se uma tendência de estabilidade. Houve ganho de apenas uma espécie no componente adulto, a Acca sellowiana (O.Berg) Burret., e de três espécies no componente regenerante: Myrceugenia pilotantha (Kiaersk.) Landrum, Xylosma tweediana (Clos) Eichler e Eugenia sp. Dessa forma, infere-se que, na trajetória sucessional no período de estudo do remanescente avaliado, o ganho estrutural em número de indivíduos e em área basal foi mais veloz do que o ganho do número de espécies. Esse é um aspecto esperado, pois, como demonstrado por Liebsch, Marques e Goldenberg (2008), durante o processo de sucessão florestal, a recuperação dos 
diferentes indicadores ecológicos da vegetação não ocorre de forma uniforme e na mesma velocidade. Além disso, não são esperadas grandes mudanças florísticas em um curto período de avaliação, sendo recomendada a continuidade da avaliação ao longo dos anos para melhor detecção das mudanças na riqueza de espécies.

Tabela 1 - Dinâmica dos componentes adulto e regenerante nos períodos de 20112015 e 2013-2015, respectivamente, das espécies arbóreas de um fragmento de floresta nebular no Planalto Catarinense

\begin{tabular}{|c|c|c|c|c|c|c|c|c|c|c|c|}
\hline \multirow[t]{2}{*}{ Espécies } & \multirow[t]{2}{*}{ GR } & \multicolumn{3}{|c|}{$\begin{array}{l}\text { Adultos: número } \\
\text { de indivíduos }\end{array}$} & \multicolumn{3}{|c|}{ Adultos: área basal } & \multicolumn{3}{|c|}{$\begin{array}{l}\text { Regenerantes: } \\
\text { número de } \\
\text { indivíduos } \\
\end{array}$} & \multirow[t]{2}{*}{$\mathbf{R}$} \\
\hline & & $N_{1}$ & $\mathbf{N}_{2}$ & $\mathrm{ML}_{\mathrm{N}}$ & $\mathrm{AB}_{1}$ & $\mathrm{AB}_{2}$ & $\mathrm{ML}_{\mathrm{AB}}$ & $\mathrm{N}_{1}$ & $\mathrm{~N}_{2}$ & $\mathrm{ML}_{\mathrm{N}}$ & \\
\hline $\begin{array}{l}\text { Acca sellowiana } \\
\text { (O.Berg) Burret }\end{array}$ & $P$ & - & 1 & 0,0 & - & 0,005 & 0,0 & - & - & - & 5511 \\
\hline $\begin{array}{l}\text { Araucaria angustifolia } \\
\text { (Bertol.) Kuntze }\end{array}$ & CEL & 8 & 8 & 0,0 & 0,185 & 0,197 & 6,3 & 4 & 4 & 0,0 & - \\
\hline Bacharis sp. & - & 2 & 1 & $-50,0$ & 0,006 & 0,003 & $-48,5$ & - & - & - & 5524 \\
\hline $\begin{array}{l}\text { Berberis laurina Billb. } \\
\text { Blepharocalyx }\end{array}$ & $\mathrm{P}$ & 2 & 3 & 50,0 & 0,007 & 0,011 & 54,0 & 2 & 5 & 150,0 & 5520 \\
\hline $\begin{array}{l}\text { salicifolius (Kunth) } \\
\text { O.Berg }\end{array}$ & CEL & 1 & 1 & 0,0 & 0,075 & 0,085 & 12,4 & - & - & - & - \\
\hline $\begin{array}{l}\text { Cinnamomum } \\
\text { amoenum (Nees \& } \\
\text { Mart.) Kosterm. }\end{array}$ & CEL & 38 & 36 & $-5,3$ & 1,827 & 1,879 & 2,9 & - & - & - & 5521 \\
\hline $\begin{array}{l}\text { Citronella paniculata } \\
\text { (Mart.) R.A.Howard } \\
\text { Dasyphyllum }\end{array}$ & CEL & 5 & 5 & 0,0 & 0,123 & 0,132 & 7,2 & 13 & 11 & $-15,4$ & 5519 \\
\hline $\begin{array}{l}\text { brasiliense (Spreng.) } \\
\text { Cabrera }\end{array}$ & $P$ & - & - & - & - & - & - & 2 & 1 & $-50,0$ & - \\
\hline $\begin{array}{l}\text { Dicksonia sellowiana } \\
\text { Hook. }\end{array}$ & CTS & 108 & 106 & $-1,9$ & 5,758 & 6,061 & 5,3 & 1 & 1 & 0,0 & - \\
\hline $\begin{array}{l}\text { Drimys } \\
\text { angustifolia Miers }\end{array}$ & CEL & 90 & 98 & 8,9 & 1,391 & 1,533 & 10,2 & 87 & 98 & 12,6 & 5522 \\
\hline $\begin{array}{l}\text { Escallonia bifida Link } \\
\text { \& Otto }\end{array}$ & $\mathrm{P}$ & 5 & 4 & $-20,0$ & 0,105 & 0,108 & 2,4 & - & - & - & 5510 \\
\hline Eugenia pluriflora DC. & CEL & - & - & - & - & - & - & 1 & 1 & 0,0 & - \\
\hline $\begin{array}{l}\text { Eugenia sp. } \\
\text { Moquiniastrum }\end{array}$ & - & 1 & 1 & 0,0 & 0,008 & 0,009 & 9,6 & - & 3 & 0,0 & 5514 \\
\hline $\begin{array}{l}\text { polymorphum (Less.) } \\
\text { G. Sancho }\end{array}$ & $P$ & - & - & - & - & - & - & 1 & 1 & 0,0 & - \\
\hline
\end{tabular}


Tabela 1 - Continuação

\begin{tabular}{|c|c|c|c|c|c|c|c|c|c|c|c|}
\hline \multirow[t]{2}{*}{ Espécies } & \multirow[t]{2}{*}{ GR } & \multicolumn{3}{|c|}{$\begin{array}{l}\text { Adultos: número } \\
\text { de indivíduos }\end{array}$} & \multicolumn{3}{|c|}{ Adultos: área basal } & \multicolumn{3}{|c|}{$\begin{array}{c}\text { Regenerantes: } \\
\text { número de } \\
\text { indivíduos }\end{array}$} & \multirow[t]{2}{*}{$\mathbf{R}$} \\
\hline & & $N_{1}$ & $\mathbf{N}_{2}$ & $\mathrm{ML}_{\mathrm{N}}$ & $\mathrm{AB}_{1}$ & $\mathrm{AB}_{2}$ & $\mathrm{ML}_{\mathrm{AB}}$ & $\mathbf{N}_{1}$ & $\mathbf{N}_{2}$ & $\mathrm{ML}_{\mathrm{N}}$ & \\
\hline $\begin{array}{l}\text { Ilex theezans Mart. ex } \\
\text { Reissek }\end{array}$ & CEL & - & - & - & - & - & - & 1 & 2 & 100,0 & - \\
\hline $\begin{array}{l}\text { Ilex microdonta } \\
\text { Reissek }\end{array}$ & CTS & 277 & 278 & 0,4 & 10,386 & 10,872 & 4,7 & 24 & 24 & 0,0 & 5513 \\
\hline $\begin{array}{l}\text { Ilex paraguariensis } \\
\text { A.St.-Hil. }\end{array}$ & CTS & 13 & 12 & $-7,7$ & 0,360 & 0,369 & 2,6 & 10 & 6 & $-40,0$ & 5512 \\
\hline $\begin{array}{l}\text { Maytenus } \\
\text { boaria Molina }\end{array}$ & CEL & 43 & 44 & 2,3 & 0,901 & 0,961 & 6,7 & 3 & 2 & $-33,3$ & 5540 \\
\hline $\begin{array}{l}\text { Myrceugenia euosma } \\
\text { (O.Berg) D.Legrand }\end{array}$ & CEL & 510 & 509 & $-0,2$ & 13,707 & 14,273 & 4,1 & 21 & 26 & 23,8 & 5527 \\
\hline $\begin{array}{l}\text { Myrceugenia } \\
\text { glaucescens (Cambess.) } \\
\text { D.Legrand \& Kausel }\end{array}$ & CEL & 19 & 24 & 26,3 & 0,089 & 0,116 & 30,4 & 19 & 20 & 5,3 & 5533 \\
\hline $\begin{array}{l}\text { Myrceugenia miersiana } \\
\text { (Gardner) D.Legrand \& } \\
\text { Kausel }\end{array}$ & CEL & 28 & 29 & 3,6 & 0,125 & 0,126 & 1,2 & 50 & 75 & 50,0 & 5537 \\
\hline Myrceugenia & & & & & & & & & & & \\
\hline $\begin{array}{l}\text { myrcioides (Cambess.) } \\
\text { O.Berg }\end{array}$ & CEL & 16 & 16 & 0,0 & 0,172 & 0,173 & 0,4 & 2 & 1 & $-50,0$ & 5530 \\
\hline Myrceugenia & & & & & & & & & & & \\
\hline $\begin{array}{l}\text { regnelliana (O.Berg) D. } \\
\text { Legrand \& Kausel }\end{array}$ & CEL & 57 & 57 & 0,0 & 1,121 & 1,207 & 7,7 & 62 & 65 & 4,8 & 5538 \\
\hline $\begin{array}{l}\text { Myrceugenia oxysepala } \\
\text { (Burret) D.Legrand \& } \\
\text { Kausel }\end{array}$ & CEL & 63 & 64 & 1,6 & 0,393 & 0,398 & 1,3 & 20 & 19 & $-5,0$ & 5518 \\
\hline $\begin{array}{l}\text { Myrceugenia pilotantha } \\
\text { (Kiaersk.) Landrum }\end{array}$ & CEL & 3 & 5 & 66,7 & 0,013 & 0,014 & 5,7 & - & 7 & 0,0 & 5515 \\
\hline $\begin{array}{l}\text { Myrcia hatschbachii } \\
\text { D.Legrand }\end{array}$ & CEL & - & - & - & - & - & - & 1 & 1 & 0,0 & - \\
\hline $\begin{array}{l}\text { Myrcia hartwegiana (O. } \\
\text { Berg) Kiaersk. }\end{array}$ & CEL & 1 & 1 & 0,0 & 0,003 & 0,005 & 66,3 & - & - & - & - \\
\hline $\begin{array}{l}\text { Myrrhinium } \\
\text { atropurpureum Schott }\end{array}$ & CEL & 19 & 21 & 10,5 & 0,176 & 0,220 & 25,0 & 3 & 3 & 0,0 & 5535 \\
\hline Myrsine coriacea ((Sw.) & & & & & & & & & & & \\
\hline $\begin{array}{l}\text { R.Br. ex Roem. \& } \\
\text { Schult. }\end{array}$ & CEL & 19 & 18 & $-5,3$ & 0,300 & 0,322 & 7,1 & 11 & 15 & 36,4 & 5531 \\
\hline $\begin{array}{l}\text { Myrsine umbellata } \\
\text { Mart. }\end{array}$ & CEL & - & - & - & - & - & - & 2 & 2 & 0,0 & - \\
\hline
\end{tabular}




\begin{tabular}{|c|c|c|c|c|c|c|c|c|c|c|c|}
\hline \multirow[t]{2}{*}{ Espécies } & \multirow[t]{2}{*}{ GR } & \multicolumn{3}{|c|}{$\begin{array}{l}\text { Adultos: número } \\
\text { de indivíduos }\end{array}$} & \multicolumn{3}{|c|}{ Adultos: área basal } & \multicolumn{3}{|c|}{$\begin{array}{l}\text { Regenerantes: } \\
\text { número de } \\
\text { indivíduos }\end{array}$} & \multirow[t]{2}{*}{$\mathbf{R}$} \\
\hline & & $\mathbf{N}_{1}$ & $\mathbf{N}_{2}$ & $\mathrm{ML}_{\mathrm{N}}$ & $\mathrm{AB}_{1}$ & $\mathrm{AB}_{2}$ & $M L_{A B}$ & $\mathbf{N}_{1}$ & $\mathbf{N}_{2}$ & $\mathrm{ML}_{\mathrm{N}}$ & \\
\hline $\begin{array}{l}\text { Ocotea pulchella (Nees } \\
\text { \& Mart.) Mez }\end{array}$ & CEL & 69 & 66 & $-4,4$ & 1,400 & 1,392 & $-0,6$ & 28 & 31 & 10,7 & 5539 \\
\hline $\begin{array}{l}\text { Persea willdenowii } \\
\text { Kosterm. (CEL) }\end{array}$ & CEL & 5 & 4 & $-20,0$ & 0,051 & 0,054 & 4,5 & - & - & - & 5536 \\
\hline $\begin{array}{l}\text { Prunus myrtifolia (L.) } \\
\text { Urb. }\end{array}$ & CEL & 83 & 85 & 2,4 & 1,534 & 1,628 & 6,0 & 13 & 16 & 23,1 & 5517 \\
\hline $\begin{array}{l}\text { Rhamnus } \\
\text { sphaerosperma SW. }\end{array}$ & CEL & 2 & 1 & $-50,0$ & 0,014 & 0,013 & $-3,0$ & - & - & - & 5526 \\
\hline $\begin{array}{l}\text { Schinus polygamus } \\
\text { (Cav.) Cabrera }\end{array}$ & $P$ & 20 & 20 & 0,0 & 0,159 & 0,161 & 1,6 & 2 & 2 & 0,0 & 5532 \\
\hline $\begin{array}{l}\text { Solanum pabstii } \\
\text { L.B.Sm. \& Downs }\end{array}$ & CEL & 1 & 1 & 0,0 & 0,003 & 0,006 & 68,2 & - & - & - & 5523 \\
\hline $\begin{array}{l}\text { Styrax leprosus Hook. } \\
\text { \& Arn. }\end{array}$ & CEL & 3 & 3 & 0,0 & 0,081 & 0,087 & 6,6 & - & - & - & 5525 \\
\hline $\begin{array}{l}\text { Symphyopappus } \\
\text { itatiayensis (Hieron.) } \\
\text { R.M.King \& H.Rob. }\end{array}$ & $P$ & - & - & - & - & - & - & 131 & 147 & 12,2 & - \\
\hline $\begin{array}{l}\text { Symphyopappus cf. } \\
\text { lymansmithii B.L. Rob. } \\
\text { (P) }\end{array}$ & $P$ & 2 & 1 & $-50,0$ & 0,004 & 0,003 & $-43,3$ & - & - & - & 5529 \\
\hline $\begin{array}{l}\text { Symplocos cf. tetrandra } \\
\text { Mart. }\end{array}$ & CEL & 29 & 29 & 0,0 & 0,565 & 0,631 & 11,7 & 3 & 1 & $-66,7$ & 5516 \\
\hline $\begin{array}{l}\text { Symplocos uniflora } \\
\text { (Pohl) Benth. }\end{array}$ & CEL & - & - & - & - & - & - & 9 & 13 & 44,4 & - \\
\hline $\begin{array}{l}\text { Xylosma tweediana } \\
\text { (Clos) Eichler (CEL) }\end{array}$ & CEL & - & - & - & - & - & - & - & 1 & 0,0 & - \\
\hline $\begin{array}{l}\text { Weinmannia } \\
\text { paulliniifolia Puhl ex } \\
\text { Ser. }\end{array}$ & CEL & 40 & 39 & $-2,5$ & 0,900 & 0,977 & 8,5 & - & - & - & 5534 \\
\hline Total de espécies & & 33 & 34 & - & - & - & - & 28 & 31 & - & - \\
\hline Total de indivíduos & & 1582 & 1591 & 0,6 & 41,944 & 44,028 & 5,0 & 526 & 604 & 14,8 & - \\
\hline
\end{tabular}

Fonte: Autores (2021)

Em que: $\mathrm{GR}$ = guildas de regeneração, sendo $\mathrm{P}$ = pioneira; $\mathrm{CEL}$ = clímax exigente de luz; CTS = clímax tolerante à sombra; $N_{1}=$ número de indivíduos inicial (2011 para o componente adulto e 2013 para o regenerante); $\mathrm{N}_{2}$ = número de indivíduos final (2015); $\mathrm{ML}_{\mathrm{N}}=$ mudança líquida em número de indivíduos $\left(\%\right.$.ano-1); $A B_{1}$ = área basal inicial $\left(2011\right.$, em m².ha-1); $A B_{2}=$ área basal final $\left(2015\right.$, em m$^{2}$.ha-1); $M L A B=$ mudança líquida em área basal $\left(\% \cdot a n 0^{-1}\right) . R$ = número de registro no Herbário LUSC, da Universidade do Estado de Santa Catarina (UDESC). 
Em ambos os componentes, em todos os anos de avaliação, ocorreu a predominância de espécies e de indivíduos classificados como clímax exigente em luz (CEL) (em torno de 70 \% para todos os casos) (Tabela 2), não havendo alterações das proporções das guildas ao longo do tempo $(p>0,3)$. Destaca-se que a principal diferença entre os dois componentes se refere à representatividade de indivíduos pertencentes a espécies pioneiras (P) e climácicas tolerantes ao sombreamento (CTS). Para os adultos, depois das CEL, as CTS se destacaram, com 25,21 \% (2011) e 24,92 \% (2015) dos indivíduos. Já para os regenerantes, as P substituíram as CTS como o segundo grupo de maior representatividade de indivíduos, com 26,24 \% e 25,96 \% para, respectivamente, 2013 e 2015.

Tabela 2 - Teste de proporção para a riqueza e a abundância das diferentes guildas de regeneração das espécies arbóreas de um fragmento de floresta nebular no Planalto Catarinense. Valores entre parênteses indicam a proporção da abundância e riqueza para os diferentes anos avaliados, sendo o ano 1, 2011 para os adultos e 2013 para os regenerantes, e o ano 2, 2015 para ambos os componentes

\begin{tabular}{|c|c|c|c|c|c|c|}
\hline & \multicolumn{3}{|c|}{$\begin{array}{c}\text { Número de espécies } \\
\text { (riqueza) }\end{array}$} & \multicolumn{3}{|c|}{$\begin{array}{c}\text { Número de indivíduos } \\
\text { (abundância) }\end{array}$} \\
\hline & \multicolumn{3}{|c|}{ Adultos } & \multicolumn{3}{|c|}{ Adultos } \\
\hline & Ano 1 & Ano 2 & $p$ & Ano 1 & Ano 2 & $p$ \\
\hline$P$ & $4(12,90)$ & $5(15,63)$ & 1 & $29(1,84)$ & $29(1,83)$ & 1 \\
\hline CEL & $24(77,42)$ & $24(75,00)$ & 1 & $1152(72,96)$ & $1164(73,25)$ & 0,8825 \\
\hline \multirow[t]{3}{*}{ CTS } & $3(9,68)$ & $3(9,38)$ & 1 & $398(25,21)$ & $396(24,92)$ & 0,8857 \\
\hline & \multicolumn{3}{|c|}{ Regenerantes } & \multicolumn{3}{|c|}{ Regenerantes } \\
\hline & Ano 1 & Ano 2 & $\mathrm{p}$ & Ano 1 & Ano 2 & $p$ \\
\hline$P$ & $5(17,86)$ & $5(16,67)$ & 1 & $138(26,24)$ & $156(25,96)$ & 0,9693 \\
\hline CEL & $20(71,43)$ & $22(73,33)$ & 1 & $353(67,11)$ & $414(68,89)$ & 0,5663 \\
\hline CTS & $3(10,71)$ & $3(10,00)$ & 1 & $35(6,65)$ & $31(5,16)$ & 0,3473 \\
\hline
\end{tabular}

Fonte: Autores (2021)

Em que: $\mathrm{P}$ = pioneiras; $\mathrm{CEL}$ = clímax exigente de luz; $\mathrm{CTS}$ = clímax tolerante à sombra.

A representatividade das guildas de regeneração em relação às espécies e aos indivíduos é um importante indicador do estágio sucessional em que se encontra uma 
floresta. Liebsch et al. (2008), em uma meta-análise de cronossequências em áreas de Floresta Atlântica em sua porção sul, verificaram que florestas que apresentavam um percentual de espécies não pioneiras ( 85 a $87 \%$ ), semelhante ao observado no presente estudo, possuíam uma idade de 60-80 anos pós-distúrbio. Isso indica que, apesar dos impactos que a floresta esteve sujeita no passado, esta se encontra em estágio sucessional avançado. Por outro lado, para o componente regenerante, a elevada representatividade de indivíduos pertencentes a espécies pioneiras não é um padrão comum em florestas que se encontram em estágio sucessional final, uma vez que, nessas circunstâncias, espera-se um dossel mais fechado, favorecendo o estabelecimento de espécies tolerantes ao sombreamento. Por isso, os resultados encontrados sugerem que a floresta passou por perturbações recentes, que resultaram na abertura de clareiras, favorecendo o estabelecimento de espécies pioneiras no componente regenerante. Como Marcon et al. (2014) observaram, no ano de 2011, um dossel predominantemente fechado na área, é possível inferir que o surgimento de clareiras tenha ocorrido pouco tempo antes do primeiro levantamento do componente regenerativo, que foi realizado somente em 2013. Sevegnani et al. (2012) observaram, em seus estudos na Bacia do Rio Pelotas - o que inclui a área de estudo -, que impactos antrópicos são comuns na região, especialmente a exploração seletiva de espécies de interesse e o pastejo pelo gado nos remanescentes. No presente estudo, dentre as espécies pioneiras favorecidas nesse processo de abertura de clareiras, destacouse Symphyopappus itatiayensis (Hieron.) R.M.King \& H.Rob., que foi a mais abundante na regeneração natural, com um aumento no tamanho populacional de $12,2 \%$.ano-1, porém ausente no componente adulto.

Ao se analisar as taxas demográficas das 10 espécies mais abundantes no componente adulto no primeiro levantamento, observa-se que cinco apresentaram ganho em número de indivíduos: Ilex microdonta Reissek; Drimys angustifolia Miers; Prunus myrtifolia (L.) Urb.; Myrceugenia oxysepala (Burret) D.Legrand \& Kausel, e Maytenus boaria Molina. Quatro espécies apresentaram redução no tamanho 
populacional: Myrceugenia euosma (O.Berg) D.Legrand; Dicksonia sellowiana Hook.; Ocotea pulchella (Nees \& Mart.) Mez, e Weinmannia paulliniifolia Puhl ex Ser. Note-se que uma espécie permaneceu estável, a Myrceugenia regnelliana (O.Berg) D. Legrand \& Kausel. Quanto à área basal, com exceção de Ocotea pulchella, todas as citadas acima apresentaram ganho, confirmando o padrão de incremento na área estudada.

Em relação às 10 espécies mais abundantes do componente regenerativo em 2013, oito apresentaram incremento: Symphyopappus itayaensis; Drimys angustifolia; Myrceugenia regnelliana; Myrceugenia miersiana (Gardner) D.Legrand \& Kausel; Ocotea pulchella; Myrceugenia euosma; Myrceugenia glaucescens (Cambess.) D.Legrand \& Kausel, e Prunus myrtifolia. Uma espécie apresentou redução, a Myrceugenia oxysepala, e uma mostrou estabilidade, a llex microdonta, no tamanho populacional, também confirmando a predominância de aumento populacional.

Destacaram-se também, entre as espécies mais abundantes em ambos os componentes, Drimys angustifolia e Prunus myrtifolia, que apresentaram ganhos em todos os aspectos demográficos avaliados, demonstrando que apresentam elevado potencial regenerativo e encontram-se bem adaptadas à floresta. O fato de ambas as espécies serem classificadas como exigentes em luz reforça a ideia de que as condições ambientais do fragmento estejam favorecendo o estabelecimento de espécies pertencentes a esta guilda de regeneração. Drimys angustifolia, espécie típica das florestas nebulares da região, pode ser recomendada, portanto, para a recomposição de florestas degradadas nesses ambientes. Além de esta apresentar bom crescimento e regeneração nas condições ambientais estudadas, fornece fruto de interesse para a fauna, o que irá atrair dispersores para áreas em recuperação. O mesmo acontece com os frutos de Prunus myrtifolia, espécie que Longhi et al. (2006) também encontraram como de elevado incremento diamétrico e, segundo Avila et al. (2011), pode ser utilizada também para a recomposição de florestas ciliares. 


\section{CONCLUSÕES}

Foi possível concluir que a dinâmica de ambos os componentes (adulto e regenerante) da floresta nebular estudada indicou uma área em estruturação, com destaque para o ganho em área basal do componente adulto e o incremento na abundância do componente regenerativo. A maioria das espécies e dos indivíduos foi classificada como clímax exigentes em luz em ambos os componentes e, apesar das representatividades das diferentes guildas de regeneração não terem se alterado no período avaliado, os resultados sobre a demografia das espécies indicam o favorecimento da regeneração de espécies exigentes em luz, que são comuns em florestas em fases mais iniciais de sucessão. A ocorrência de distúrbios recentes, resultando na formação de clareiras e/ou perturbações crônicas observadas na área, como a presença de gado, pode explicar esses resultados observados. Destaca-se, ainda, que, de médio a longo prazo, o processo de sucessão poderá retornar a uma fase mais inicial, tendo em vista a possibilidade de substituição das árvores que hoje ocupam o dossel por aquelas classificadas como pioneiras, que ocupam de forma abundante o sub-bosque.

Recomenda-se maior proteção das florestas na região, com o cercamento dos remanescentes, objetivando maximizar a conservação das áreas. Além disso, é necessário, também, o acompanhamento da dinâmica a fim de determinar se mudanças sucessionais ocorrerão em intervalo de tempo maior, o que poderá contribuir para um maior entendimento do funcionamento da dinâmica em florestas nebulares.

\section{AGRADECIMENTOS}

Ao CNPq pela bolsa de pesquisa ao primeiro autor e pelas bolsas de produtividade concedidas ao segundo e terceiro autores. À FAPESC pelo apoio financeiro por meio do Edital PAP/FAPESC/UDESC. Ao Dr. Marcos Eduardo Guerra Sobral pela identificação das espécies da família Myrtaceae. 


\section{REFERÊNCIAS}

AVILA, A.L. de et al. Caracterização da vegetação e espécies para recuperação de mata ciliar, Ijuí, RS. Ciência Florestal, Santa Maria, v. 21, n. 2, p. 251-260, 2011.

CARVALHO, M. M. X.; NODARI, E. S. As origens da indústria madeireira e do desmatamento da floresta de araucária no Médio Vale do Iguaçu (1884-1920). Cadernos do CEOM, Chapecó, v. 21, n. 29, p. 63-82, 2008.

BERTONCELLO, R.; YAMAMOTO, K.; MEIRELES, L. D.; SHEPHERD, G. J. A phytogeographic analysis of cloud forests and other forest subtypes amidst the Atlantic forests in south and southeast Brazil. Biodiversity and Conservation, Nova York, v. 20, n. 14., 3413-3433, 2011.

DALLA ROSA, A. et al. Natural regeneration of tree species in a cloud forest in Santa Catarina, Brazil. Revista Árvore, Viçosa, v. 40, n. 6, p. 1083-1092, 2016.

FERREIRA, T. Atividade madeireira no meio oeste catarinense e a Vila de Campinha Redonda. Revista Santa Catarina em História, Florianópolis, v. 1, n. 2, p. 59-65, 2009.

FORMENTO, S.; SCHORN, L. A.; RAMOS, R. A. Dinâmica estrutural arbórea de uma Floresta Ombrófila Mista em Campo Belo do Sul. Cerne, Lavras, v. 10, n. 2, p. 196-212, 2004.

GROSS, A. et al. Fragmentation as a key driver of tree community dynamics in mixed subtropical evergreen forests in Southern Brazil. Forest Ecology and Management, Amsterdam, v. 411, n. 1, 20-26, 2018.

HAMILTON, L. S.; JUVIK, J. O.; SCATENA, F. N. The Puerto Rico tropical cloud forest symposium: introduction and workshop synthesis. In: HAMILTON, L. S.; JUVIK, J. O.; SCATENA, F. N. (Eds.). Tropical montane cloud forests. New York: Springer Verlag, 1995, p.1-23.

HIGUCHI, P. et al. Dinâmica da comunidade arbórea em um fragmento de floresta estacional semidecidual montana em Lavras, Minas Gerais, em diferentes classes de solos. Revista Árvore, Viçosa, v. 32, n. 3, p. 417-426, 2008.

HIGUCHI, P. et al. Floristic composition and phytogeography of the tree component of Araucaria Forest fragments in southern Brazil. Brazilian Journal of Botany, São Paulo, v. 35, n. 2, p. 145157, 2012.

IBGE. Manual técnico da vegetação brasileira. Rio de Janeiro: Fundação Instituto Brasileiro de Geografia e Estatística, 2012. 271 p.

KORNING, J.; BALSLEV, H. Growth and mortality of trees in Amazonian tropical rain forest in Ecuador. Journal of Vegetation Science, Hoboken, v. 4, n. 1, p. 77-86, 1994.

LEITE, E. C.; RODRIGUES, R. R. Análise do mosaico silvático em um fragmento de floresta tropical estacional no sudeste do Brasil. Revista Árvore, Viçosa, v. 32, n. 3, p. 443-452, 2008.

LEYSER, G. et al. Regeneração de espécies arbóreas e relações com o componente adulto em uma floresta estacional no vale do rio Uruguai, Brasil. Acta Botanica Brasilica, Belo Horizonte, v. 26, n. 1, p. 74-83, 2012. 
LIEBERMAN, D.; LIEBERMAN, M. Forest tree growth and dynamics at La Selva, Costa Rica (19691982). Journal of Tropical Ecology, Cambridge, v. 3, n. 3, p. 347-358, 1987.

LIEBSCH, D.; MARQUES, M. C. M.; GOLDENBERG, R. How long does the Atlantic Rain Forest take to recover after a disturbance? Changes in species composition and ecological features in the secondary succession. Biological Conservation, Amsterdam, v. 141, n. 6, p. 1717-1725, 2008.

LINDENMAIER, D. S.; BUDKE, J. C. Florística, diversidade e distribuição espacial das espécies arbóreas em uma floresta estacional na bacia do rio Jacuí, Sul do Brasil. Pesquisas Botânicas, São Leopoldo, s/v, n. 57, p. 193-216, 2006.

LONGHI, L. J. et al. Classificação e caracterização de estágios sucessionais em remanescentes de Floresta Ombrófila Mista na Flona de São Francisco de Paula, RS, Brasil. Ciência Florestal, Santa Maria, v. 16, n. 2, p. 113-125, 2006.

MARCON, A. K. et al. Variação florístico-estrutural em resposta à heterogeneidade ambiental em uma Floresta Nebular em Urubici, Planalto Catarinense. Scientia Forestalis, Piracicaba, v. 42, n. 103, p. 439-450, 2014.

OLIVEIRA FILHO, A. T. et al. Effects of soils and topography on the distribution of tree species in a tropical riverine forest in south-eastern Brazil. Journal of Tropical Ecology, Cambridge, v. 10, n. 4, p. 483-508, 1994.

R DEVELOPMENT CORE TEAM. R: A language and environment for statistical computing. Disponível em: http://www.r-project.org. Acesso em: 8 de fevereiro de 2017.

SALAMI, B. et al. Influência de variáveis ambientais na dinâmica do componente arbóreo em um fragmento de Floresta Ombrófila Mista em Lages, SC. Scientia Forestalis, Piracicaba, v. 42, n. 102, p. 197-207, 2014.

SANQUETTA, C. R.; DALLA CORTE, A. P.; EISFELD, R. L. Crescimento, mortalidade e recrutamento em duas florestas de araucária (Araucaria angustifolia (Bert.) O. Ktze.) no Estado do Paraná, Brasil. Revista Ciências Exatas e Naturais, Guarapuava, v. 5, n. 1, p. 101-112, 2003.

SANTOS, V.S. et al. Dinâmica florestal de espécies arbóreas em uma floresta de várzea na cidade de Macapá, AP, Brasil. Revista Verde de Agroecologia e Desenvolvimento Sustentável, Pombal, v. 7, n. 4, p. 207-213, 2012.

SANTOS, K. F. et al. Regeneração natural do componente arbóreo após a mortalidade de um maciço de taquara em um fragmento de floresta ombrófila mista em Lages - SC. Ciência Florestal, Santa Maria, v. 25, n. 1, p. 107-117, 2015.

SCHEER, M. B., MOCOCHINSK, A. Y. Florística vascular da Floresta Ombrófila Densa Altomontana de quatro serras no Paraná. Biota Neotropica, Campinas, v. 9, n. 2, p. 51-70, 2009.

SEVEGNANI, L. et al. Flora arbórea e o impacto humano nos fragmentos florestais na Bacia do Rio Pelotas, Santa Catarina, Brasil. Revista de Estudos Ambientais, Blumenau, v. 14, n. 1, p. 60-73, 2012. 
SCHEIL, D.; JENNINGS, S.; SAVILL, P. Long-term permanent plot observations of vegetation dynamics in Bundongo, a Ugandan rain forest. Journal of Tropical Ecology, Cambridge, v. 16, n. 6, p. 675-800, 2000.

SWAINE, M. D.; WHITMORE, T. C. On the definition of ecological species groups in tropical rain forests. Vegetatio, Dordrecht, v. 75, n. 1, p. 81-86, 1988.

VEFAGO, M. B. et al. What explains the variation on the regenerative component dynamics of Araucaria Forests in southern Brazil? Scientia Agricola, Piracicaba, v. 76, n. 5, 405-414, 2019.

VOLPATO, M. M. L. Regeneração natural em uma floresta secundária no domínio de Mata Atlântica: uma análise fitossociológica. 1994. 123 f. Dissertação (Mestrado em Ciência Florestal) - Universidade Federal de Viçosa, Viçosa, 1994.

\section{Contribuição de Autoria}

\section{1 - Guilherme Neto Santos}

Engenheiro Florestal

https://orcid.org/0000-0003-0346-6941 • guineto.florestal@gmail.com

Contribuição: Investigação, Escrita - primeira versão, Escrita - revisão e edição

\section{2 - Ana Carolina da Silva}

Engenheira Florestal, Dra., Professora

https://orcid.org/0000-0002-1285-640X·carol_sil4@yahoo.com.br

Contribuição: Concepção, Recursos, Obtenção de financiamento, Metodologia, Administração do projeto, Validação, Supervisão, Escrita - revisão e edição

\section{3 - Pedro Higuchi}

Engenheiro Florestal, Dr., Professor

https://orcid.org/0000-0002-3855-555X•higuchip@gmail.com

Contribuição: Curadoria dos dados, Análise Formal, Metodologia, Software, Visualização, Escrita - revisão e edição

\section{4 - Aline Gross}

Engenheira Florestal

https://orcid.org/0000-0003-2496-8852•alinegros@hotmail.com

Contribuição: Investigação, Escrita - revisão e edição 


\section{5 - Ricardo de Vargas Kilca}

Biólogo, Dr.

https://orcid.org/0000-0003-1085-7641•ricardokilca@yahoo.com.br

Contribuição: Investigação, Escrita - revisão e edição

\section{6 - Mariele Alves Ferrer Silva}

Engenheira Florestal

https://orcid.org/0000-0003-2599-7356•mariele.ferrer@hotmail.com

Contribuição: Investigação, Escrita - revisão e edição

\section{7 - Carla Luciane Lima}

Engenheira Florestal

https://orcid.org/0000-0003-2496-8852 • carla_engflorestal@yahoo.com.br

Contribuição: Investigação, Escrita - revisão e edição

\section{8 - Aline Pereira Cruz}

Bióloga

https://orcid.org/0000-0002-9279-7293•a.line_cruz@yahoo.com.br

Contribuição: Investigação, Escrita - revisão e edição

\section{9 - Amanda da Silva Nunes}

Engenheira Florestal

https://orcid.org/0000-0001-6669-3936• amandaeng.f@gmail.com

Contribuição: Investigação, Escrita - revisão e edição

\section{0 - Vanessa Fátima Soboleski}

Engenheira Florestal

https://orcid.org/0000-0002-6817-2517 • vanessasoboleski@hotmail.com

Contribuição: Investigação, Escrita - revisão e edição

\section{Como citar este artigo}

Santos, G. N.; Silva, A. C.; Higuchi, P.; Gross, A.; Kilca, R. V.; Silva, M. A. F.; Lima, C. L.; Cruz, A. P.; Nunes, A. S.; Soboleski, V. F. Dinâmica do componente arbóreo e regenerante em uma floresta nebular no Planalto Sul Catarinense. Ciência Florestal, Santa Maria, v. 31, n. 3, p. 1086-1104, 2021. DOI 10.5902/1980509829654. Disponível em: https://doi.org/10.5902/1980509829654. Acesso em: xx mês-abreviado 2021. 